\title{
A comparison of bleeding efficiency, microbiological quality and lipid oxidation in goats subjected to conscious halal slaughter and slaughter following minimal anesthesia
}

\begin{abstract}
The study assessed the effect of conscious halal slaughter and slaughter following minimal anesthesia on bleeding efficiency of goats and keeping quality of goat meat. Ten Boer cross bucks were divided into two groups and subjected to either halal slaughter without stunning (HS) or minimal anesthesia prior to slaughter (AS). The blood lost during exsanguination was measured. Residual blood was further quantified by determination of hemoglobin and myoglobin content in longissimus lumborum muscle. Storage stability of the meat was evaluated by microbiological analysis and lipid oxidation. Blood loss at exsanguination, residual hemoglobin and lipid oxidation were not significantly different $(p>0.05)$ between HS and AS. Lactic acid bacteria was the only microbe that was significantly elevated after $24 \mathrm{~h}$ of storage at $4^{\circ} \mathrm{C}$ in the AS group. In conclusion, slaughtering goats under minimal anesthesia or fully conscious did not affect bleeding efficiency and keeping quality of goat meat.
\end{abstract}

Keyword: Bleeding efficiency; Goats; Halal slaughter; Minimal anesthesia; Storage stability 